\title{
Fabrication of periodic nanostructures using dynamic plowing lithography with the tip of an atomic force microscope
}

\author{
Yang $\mathrm{He}^{\mathrm{a}, \mathrm{b}}$, Yongda Yan ${ }^{\mathrm{a}, \mathrm{b}, *}$, Yanquan Geng ${ }^{\mathrm{a}, \mathrm{b},{ }^{*}}$ Emmanuel B. Brousseau ${ }^{\mathrm{c}}$ \\ ${ }^{a}$ The State Key Laboratory of Robotics and Systems, Robotics Institute, Harbin Institute of \\ Technology, Harbin, Heilongjiang 150080, P.R. China \\ ${ }^{b}$ Center for Precision Engineering, Harbin Institute of Technology, Harbin, Heilongjiang 150001, \\ P.R. China \\ ${ }^{c}$ Cardiff School of Engineering, Cardiff University, Cardiff, UK \\ * Corresponding author. Tel.: +86-0451-86412924. Fax: +86-0451-86415244 \\ E-mail address: yanyongda@hit.edu.cn (Y.D. Yan), gengyanquan@hit.edu.cn (Y.Q. Geng)
}

\section{ABSTRACT}

The fabrication of periodic nanostructures with a fine control of their dimensions is performed on poly(methyl methacrylate) (PMMA) thin films using an atomic force microscope technique called dynamic plowing lithography (DPL). Different scratching directions are investigated first when generating single grooves with DPL. In particular, the depth, the width and the periodicity of the machined grooves as well the height of the pile-up, formed on the side of the grooves, are assessed. It was found that these features are not significantly affected by the scratching direction, except when processing took place in a direction away from the cantilever probe and parallel to its main axis. For a given scratching direction, arrays of regular grooves are then obtained by controlling the feed, i.e. the distance between two machining lines. A scan-scratch tip trace is also used to reduce processing time and tip wear. However, irregular patterns are created when combining two layers oriented at different angles and where each layer defines an array of grooves. Thus, a "combination writing" method was implemented to fabricate arrays of grooves with a well-defined wavelength of $30 \mathrm{~nm}$, which was twice the feed value utilized. Checkerboard, diamond-shaped, and hexagonal nanodots were also fabricated. These were obtained by using the combination writing method and 
by varying the orientation and the number of layers. The density of the nanodots achieved could be as high as $1.9 \times 10^{9}$ nanodots per $\mathrm{mm}^{2}$.

Keywords: AFM, Dynamic plowing lithography (DPL), Nano-scale groove, nanodots

\section{Introduction}

Nanostructures play an important role in nanophotonic and nanoelectronic devices, especially periodic arrays of nanoscale grooves and three-dimensional (3D) nanodots. For example, Raman signal amplification occurs in shell-coated nanoparticles for surface-enhanced Raman scattering (SERS) [1]. In [2], a $500 \mathrm{~nm}$ period grating is used to guide $632.8 \mathrm{~nm}$ light on a polycarbonate substrate. In addition, a grating with varying sub-micrometre periods could be used as a photonic crystal filter with narrowband reflectivity [3]. Complex patterned nanoparticle arrays and hexagonal nanopore arrays have been shown to act as highly enhanced SERS substrates [4], and SERS is affected by nanostructure feature sizes [5]. Much effort is thus devoted to nanostructure fabrication methods. In particular, UV lithography has the advantages of cost effectiveness and mass production. However, its main limitation is in the fabrication of complex three-dimensional nanostructures such as wrinkles. Schweikart et al. reviewed fabrication methods for wrinkle patterns on surfaces [6]. When an ethanol droplet was placed on an oxidized PDMS surface, wrinkles formed in [7]. Wrinkles could be used in a microfluidic device in a liquid environment. Thin stiff films have been coated on compliant elastic substrates to produce wrinkle patterns caused by compressive stress. Various patterns have been fabricated by controlling biaxial stress, including checkerboard, hexagonal, and herringbone [8]. The wrinkle periods are determined by experimental conditions [9], and controllable-wavelength wrinkles are obtained with various forms. However, feature sizes in the sub-100 nm range, including wavelength and depth, are still difficult to obtain with this method. In contrast, focused ion beam direct writing can be used to fabricate elliptic nanostructures with $15 \mathrm{~nm}$ spacing on Si substrates as shown in [10]. However, when cost and 
production efficiency are taken into account, atomic force microscopy (AFM) tip-based nanolithography represents a better alternative. In particular, mechanical machining techniques via AFM are relatively low-cost, simple, highly accurate and provide flexible control [11]. Moreover, AFM can fabricate features on most materials.

AFM nanolithography has two modes, namely static and dynamic plowing lithography (DPL), which are derived from contact [12-17] and tapping scanning modes [18-21], respectively. In static plowing lithography, which is also known as AFM scratching, the probe tip applies a constant normal force on the surface of the sample to be processed. Lateral forces at the apex of the probe due to friction and material resistance to deformation occur during scratching. Thus, bending and torsion motions of the cantilever result from the action of such lateral forces, which is turn influence the machined depth depending on the scratching direction. In addition, the shape of the pile-up material that is accumulated on the side along each groove can be different for asymmetric tips. Wrinkle nanostructures can be fabricated by scratching grooves, which are parallel and close enough to each other. These are referred to as proximity grooves [22]. In this case, the shape of the pile-up material is combined with the shape of the grooves to define the wrinkle geometry. However, the shape, period, and depth of such nanostructures that are produced using this approach are dependent on the scratching direction, which make the process difficult to control. Nanoscale wrinkles could also be made on some polymer materials such as polycarbonate along the scratching direction defined by the tip [16]. These were referred to as nano-bundles and previous work sought solutions to control their shape and periodicity [17]. However, different parameters should be employed in each scratching direction to achieve identical depths, which makes the process more complex. Furthermore, the apex of the tip can be considerably worn after significant scratching, which can lead to a radius of about $100 \mathrm{~nm}$ [17]. Thus, the desired nanostructures can not be obtained.

In contrast to the above techniques, in the DPL method, the cantilever is driven vertically by a piezoelectric actuator like a hammer [18], and the polymer surface can be "plowed" with an 
arbitrary orientation [19]. This results in a relatively uniform groove shape and depth and thus, DPL represents a promising and reliable approach for the fabrication of complex nanostructures. More importantly, feature size can be reduced with the DPL method in comparison with static plowing lithography. The machined depth achieved with DPL is generally several nanometers, and the ridge-to-ridge width is approximately $40 \mathrm{~nm}$ [20]. A groove with a $17 \mathrm{~nm}$ mouth width [21], where the depth is measured, was obtained with optimized DPL parameters. In addition, the pile-up generated by DPL was shown to be nearly equal to the removed material in [23]. This effect can be beneficial when producing wrinkles from proximity grooves. Indeed, DPL leads to a large pile-up volume when small-depth grooves are formed by low cantilever amplitudes. Periodic nanostructures can thus be obtained via the formation of proximity grooves with the combination of the machined groove shape and the corresponding material pile-up geometry. The amplitude and periodicity of these nanostructures should be influenced by the tip trace, which includes the feed and the scratching direction. The feed corresponds to the distance between two proximity grooves. Thus, the effect of the tip trace on the resulting dimensions of an array of such grooves should be investigated further. This is the focus of the study presented here.

In this work, grooves are fabricated on a poly(methyl methacrylate) (PMMA) thin film along different predefined scratching directions for preset drive amplitudes and scratching speeds. The mouth width, period, depth, and the pile-up height are measured and analyzed for each processing direction. Arrays of grooves are fabricated with different types of tip raster scan traces. In addition, a two-step DPL method is used to fabricate checkerboard- and diamond-shaped nanodots; and hexagonal nanodots are made with a three-step method.

\section{Methods}

PMMA (molecular weight of 120000) was dissolved in chlorobenzene at a concentration of $1.25 \mathrm{wt} \%$. The solution was spun on a square single-crystal silicon substrate, which had been ultrasonically cleaned in acetone and then in alcohol for $10 \mathrm{~min}$. The spinning speed and time were 
$6000 \mathrm{rpm}$ and $30 \mathrm{~s}$, respectively, to obtain film thicknesses of several tens of nanometers. The PMMA thin film was subsequently baked at $125^{\circ} \mathrm{C}$ for $30 \mathrm{~min}$ to remove the solvent. DPL and measurement experiments were performed with a commercial AFM system (Dimension Icon; Bruker Corporation, USA). A silicon tip in tapping mode was used as the small DPL tool (MPP-11120; Bruker Corporation, USA). The scan rate was $1 \mathrm{~Hz}$ and each scan line was 256 pixels. Images were first-order flattened with the Nanoscope Analysis software provided by Bruker Company. The fabrication process was performed by the Nanoman module in the AFM system, and the Polyline command was utilized to design the tip trace for the desired grooves with arbitrary orientation.

To scratch the sample surface, the drive amplitude of the fixed cantilever end was increased to $V_{w}$ (writing voltage) as shown in Fig. 1(a). To scan to generated grooves and obtained an AFM image of their topography, the drive amplitude was reduced to $V_{r}$ (reading voltage) without changing the probe which improves the efficiency of the overall process. This was considered to be an acceptable approach because, compared with static plowing lithography, the tip wear is usually negligible for DPL. When $V_{w} / V_{r}=5$, the tip interaction with the PMMA surface was reduced, which extended the tip life. Good quality grooves with a stable machined depth were obtained with a scratching speed of $0.5 \mu \mathrm{m} / \mathrm{s}$. All experiments were performed at room temperature. 

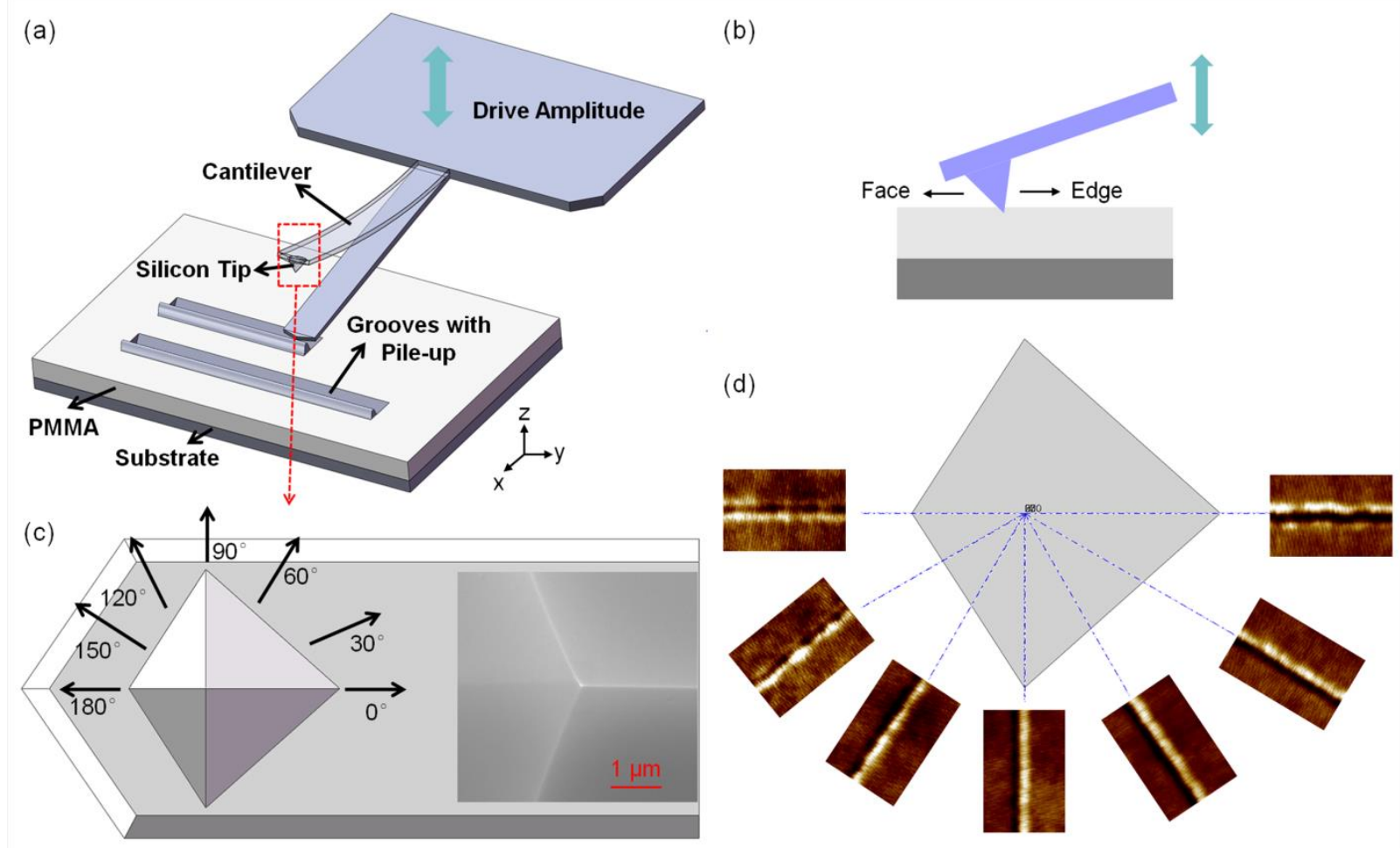

Fig1. (a) Schematic of grooves with pile-up fabrication on a PMMA thin film using DPL; (b) edgeand face-writing directions; (c) geometry of a silicon tip; (d) AFM images of grooves obtained from different scanning directions.

\section{Results and discussion}

\subsection{Effect of scratching direction}

The geometry of the silicon tip used to scratch the PMMA film is shown using a scanning electron microscopy (SEM) image in Fig. 1(c). The apex of the tip was not quite symmetrical; thus, seven scratching directions were selected in the range $0-180^{\circ}$ to simplify the experiment. The orientation angle was controlled by the PZT of the AFM system, which was preset before machining with the Nanoman module. Fig. 1(d) shows AFM images of grooves obtained at the different angles considered. At $0^{\circ}$ and $180^{\circ}$, the pile-up accumulated mainly along both sides of the grooves. For these two orientations, because of the force applied to the sample surface along the scratching direction is symmetrical due to the geometry of the tip, the material was extruded on 
both sides. However, for the other scratching directions, the pile-up accumulated along only one side.
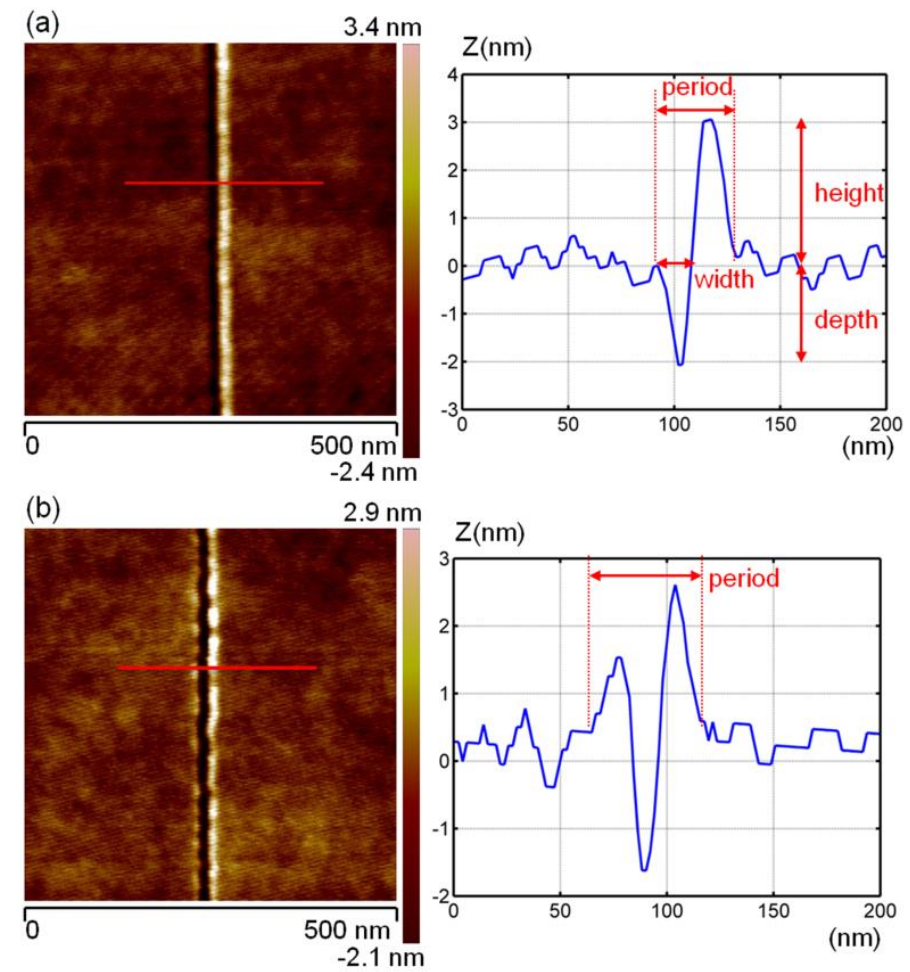

Fig. 2 Morphology and cross-sections (red lines) of grooves fabricated with (a) pile-up accumulated along one side for the $90^{\circ}$ direction, and (b) pile-up accumulated along both sides for the $0^{\circ}$ direction.

Typical groove features are shown in Fig. 2. The residual depths and the mouth width (the width at which the depth is assessed from) [24] were measured as shown in Fig. 2(a). The pile-up height was measured as well. The combined groove and pile-up widths define the period, as shown in Figs. 2(a,b). Four different types of groove dimensions are plotted in Fig. 3 as a function of the scratching angle. For all orientations, the mouth width was approximately $15 \mathrm{~nm}$ (see Fig. 3(a)), which was mainly determined by the geometry of the tip apex, which had a radius less than $10 \mathrm{~nm}$. The groove period was around $30-40 \mathrm{~nm}$ as shown in Fig. 3(b). The pile-up widths were nearly equal to the mouth widths. The groove depths were in the range of $1.5-2.0 \mathrm{~nm}$, as shown in Fig. 3(c). However, for the angle of $180^{\circ}$, the depth was lower, i.e. $1.1 \mathrm{~nm}$. The pile-up height was in 
the range 2.0-3.0 nm (see Fig. 3(d)), except, again, for the angle of $180^{\circ}$, where the height obtained was $1.0 \mathrm{~nm}$. Thus, for $180^{\circ}$, which is the face-edge scratching direction (see Fig. 1(b)), the machined depth and the pile-up height were significantly smaller than those for the grooves generated in other directions. This is likely due to the asymmetry imposed by the cantilever tilt [22]. Thus, the $180^{\circ}$ direction was avoided in the following experiments. By comparing the values of the groove depths and the pile-up heights, it can be noticed that the pile-up volume appears to be larger than the volume of removal material. When the oscillating tip penetrates the PMMA film, polymer chains are broken by the high frequency interaction [23]. This results in volume expansion, and thus the volume ratio of pile-up to the removed material is larger than one.
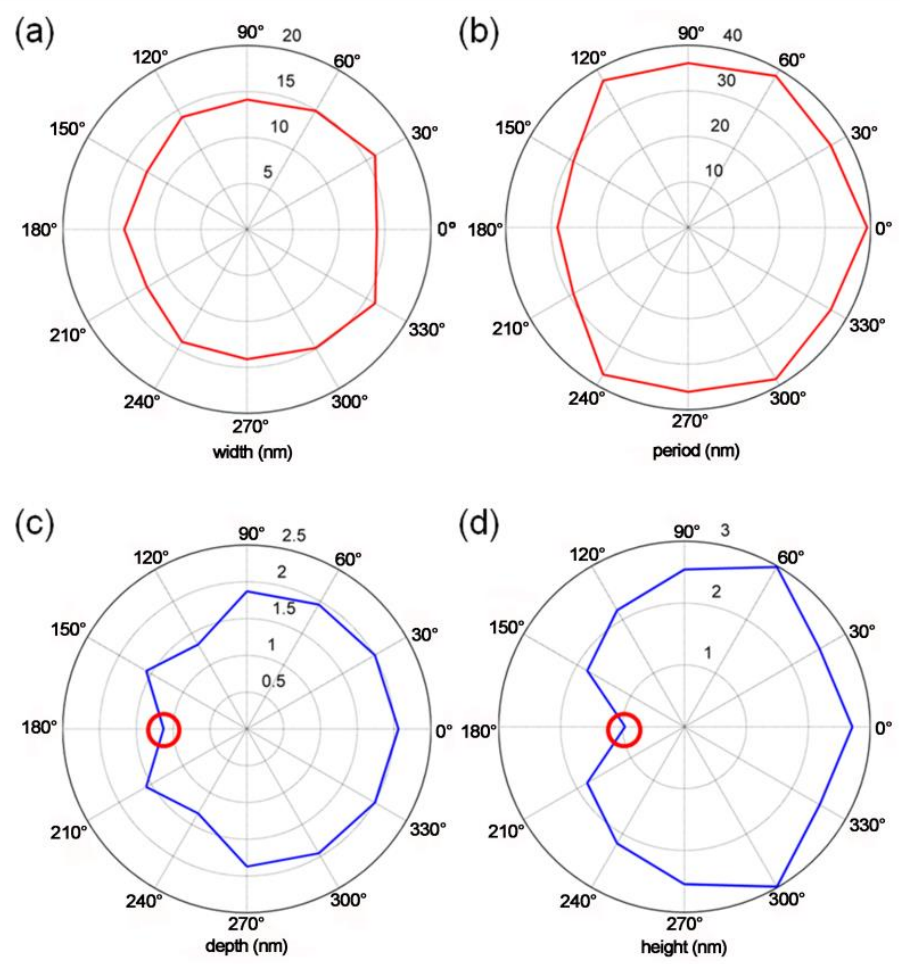

Fig. 3 Groove feature sizes: (a) width, (b) period, (c) depth, and (d) pile-up height for different scratching directions.

In the case of static plowing lithography, it is known that tip asymmetry and the deformation of the cantilever could contribute to variations in machined depth along different scratching directions [14]. Thus, it is interesting to note that grooves with a relatively uniform feature size 
were generated along all scratching directions here using the DPL approach, except for the $180^{\circ}$ angle. As an example, circles with radii of $1,2,3,4$, and $5 \mu \mathrm{m}$ were generated with DPL by defining tip traces with the Polyline command of the Nanoman software module. The AFM morphology of the obtained pattern is shown in Fig. 4(a). The depths and the mouth widths were independent on the scratching directions. In particular, the $1 \mu \mathrm{m}$ circle shown in Fig. $4(\mathrm{~b})$ is composed of continuous line patterns. The tip trace could be optimized to achieve a better fabrication result in the future. Furthermore, owing to the feature size (including depth, width, period and height), which are independent on the scratching direction with DPL, the circle pattern on a resist layer could be transferred to semiconductor materials as demonstrated in [19].

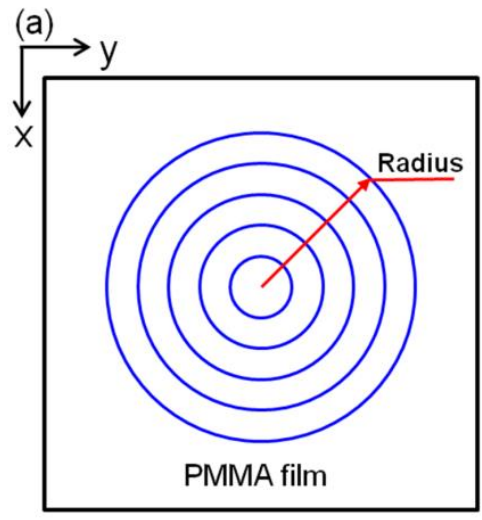

(b)

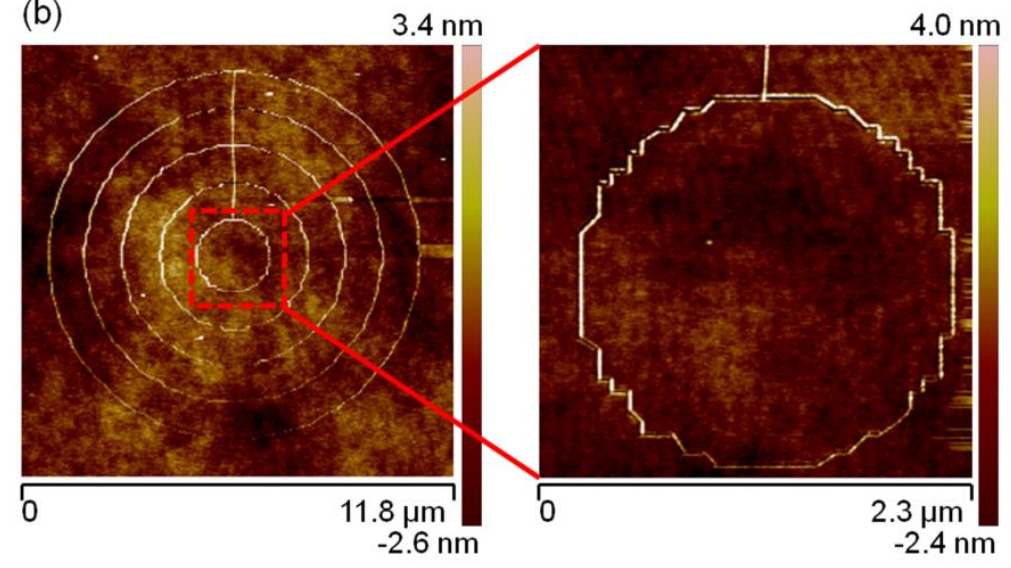

Fig. 4 (a) Schematic of tip trace, (b) morphology of circles with 1, 2, 3, 4, and $5 \mu \mathrm{m}$ radii and the inner most circle with a radius of $1 \mu \mathrm{m}$.

\subsection{Fabrication of arrays of grooves with an optimal tip trace}

Groove periods ranging from 30-40 nm are shown in Fig. 5 for feeds of $30 \mathrm{~nm}$ and $40 \mathrm{~nm}$, along with schematics of the tip traces. The scratching direction was $90^{\circ}$. At the end of each groove, $V_{w}$ was switched to $V_{r}$ to move the tip to the start of the next groove without modifying the PMMA surface. The drive amplitude was then switched back to $V_{w}$ to scratch the PMMA film. In this way, proximity grooves were fabricated with the DPL method. Fig. 5(a) shows arrays of grooves with a 
$30 \mathrm{~nm}$ feed. The wavelength was controlled by the feed, whereas, the amplitude was determined by the depth and the pile-up height, which was nearly $5 \mathrm{~nm}$ (see cross-section of Fig. 5(a)). Arrays of grooves with a feed of $40 \mathrm{~nm}$ are shown in Fig. 5(b). The wavelength and amplitude are $40 \mathrm{~nm}$ and $5 \mathrm{~nm}$, respectively. As reported in the previous section, the maximum period of a groove generated with the DPL technique here was $40 \mathrm{~nm}$. Consequently, the maximum feed which should be selected must be $40 \mathrm{~nm}$. Else, grooves may be isolated from each other and thus, the benefit of having proximity grooves would be lost. For this reason, the maximum feed utilised in this study was $40 \mathrm{~nm}$ to obtain good quality arrays of proximity grooves. The fabrication results when producing such arrays were similar for other scratching directions. The specific amplitude of the grooves was controlled by the machined depth and the pile-up height, which depended on the drive amplitude and the scratching speed. The achieved wavelength was determined by the combined width of the groove and the pile-up. The relationship between the scratching parameters and the machined depth shall be the focus of future investigations.
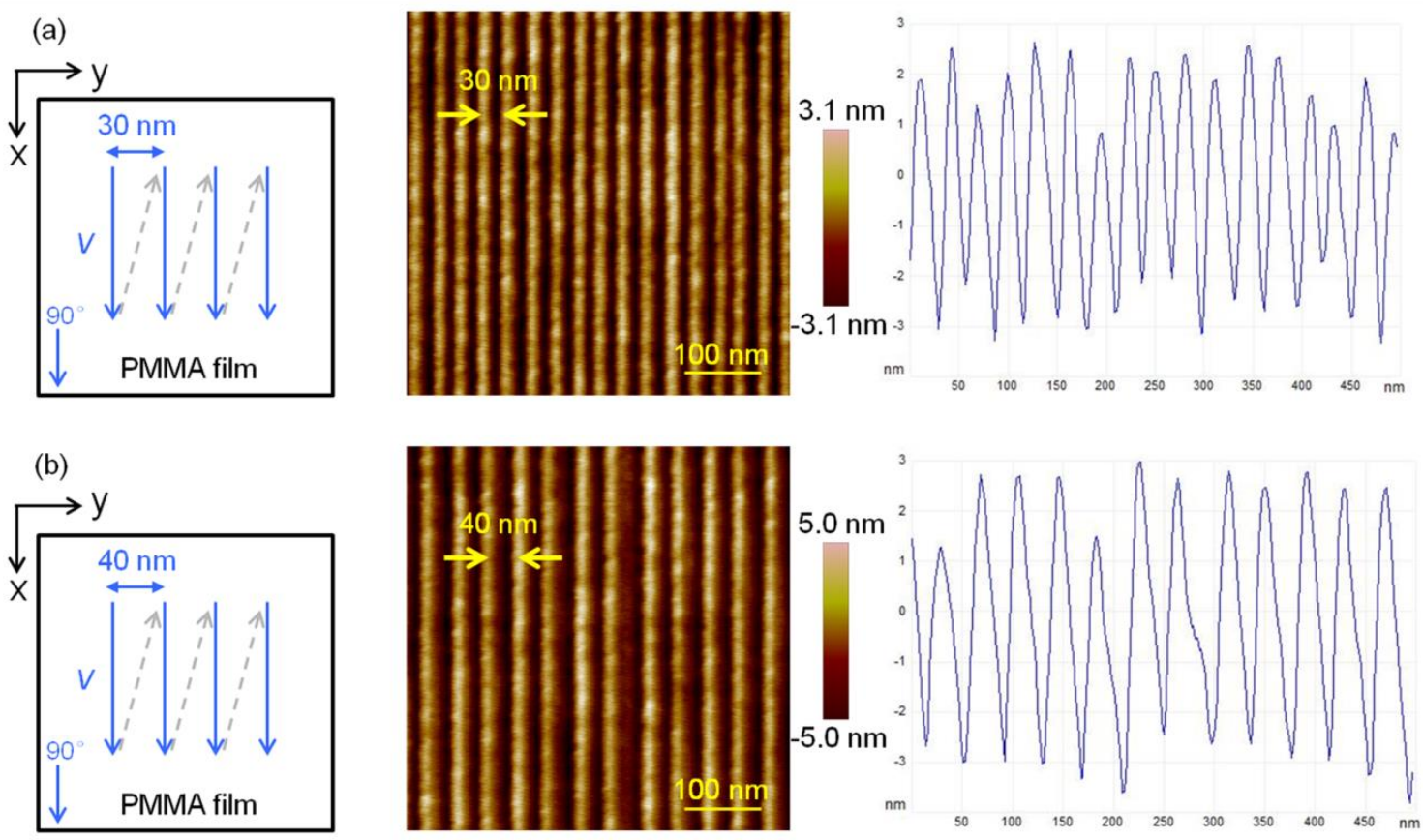

Fig. 5 Schematic of tip traces and AFM images of arrays of grooves obtained in the direction of $90^{\circ}$ for a feed of (a) $30 \mathrm{~nm}$ and (b) $40 \mathrm{~nm}$. 
Switching the drive amplitude between $V_{w}$ and $V_{r}$ during the arrays of grooves fabrication was a time-consuming process. In addition, the dynamic interaction between the tip and the PMMA surface was more complex as not constant throughout the process. A higher drive amplitude created a higher oscillation amplitude of the cantilever free end, which may cause more rapid tip wear during drive amplitude switching. Because a array of regular grooves could be obtained in any direction, a wide range of nanostructures could be generated by alternating two anti-parallel directions. Fig. 6(a) provides a schematic of such "scan-scratch" tip trace. At the starting point, the drive amplitude is $V_{w}$ to modify the PMMA surface. In this implementation, it is only when the entire tip trace is completed that the drive amplitude is switched to $V_{r}$. In this way, the probe mechanically scratches the PMMA film continuously, instead of adjusting the drive amplitude for every groove. The vertical tip trace marked in blue in Fig. 6 is oriented along the $120^{\circ}$ and $300^{\circ}$ scratching directions. From Fig. 3, it should be expected that the $300^{\circ}$ results may be similar to those of $60^{\circ}$ because of the symmetrical tip shape. Thus, this tip trace was defined as the $120^{\circ}$ scratching direction to simplify the description. For a feed of $30 \mathrm{~nm}$, the arrays of grooves in the $30^{\circ}$ and $120^{\circ}$ directions are shown in Figs. 6(b,c), respectively. A qualitative observation of the periodicity of the grooves shown in this Figure in comparison with the displayed scale bar indicates that the grooves are isolated from each other for both scratching directions. In addition, although the feature size is almost the same for each direction, the quality of the arrays of grooves was far below that obtained for scratching in just one direction. The result of a two-step scratching process is shown in Fig. 6(d). This pattern was a combination of arrays of grooves in the two scratching directions mentioned above. The feature size is independent on the scratching direction as discussed earlier. Nevertheless, non-uniform arrays of grooves were obtained with this "scan-scratch" tip trace approach. Because of the unpredicted shape of the obtained arrays (whether with proximity grooves or with isolated grooves) with the "scan-scratch" tip trace, various forms of uncontrolled patterns could be generated. 


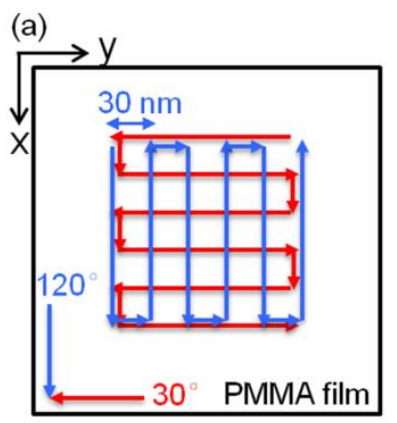

(b)

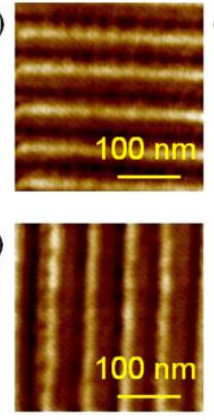

(e)

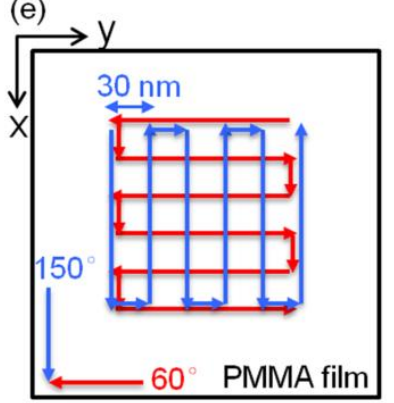

(f)

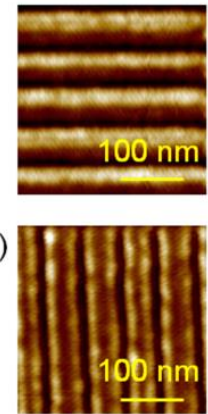

(d)

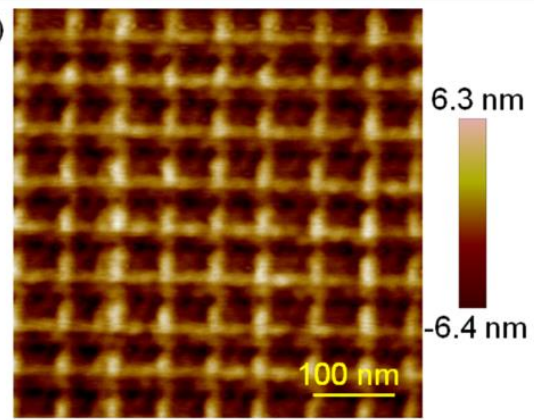

(h)

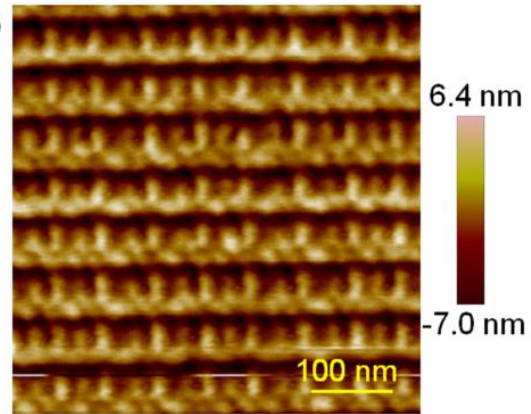

Fig. 6 Schematic of tip trace and corresponding machined results: (a) and (e) a "scan-scratch" tip trace in two pairs of anti-parallel directions. Arrays of grooves obtained with a $30 \mathrm{~nm}$ feed with a direction of (b) $30^{\circ}$ (c) $120^{\circ}$ (f) $60^{\circ}$ and (g) $150^{\circ}$. (d) and (h) Irregular patterns from the combination of arrays of grooves following a two-step DPL process.

"Combination writing" [22] was proposed to solve the problem of dissimilar pile-up in each scratching direction. This technique relies on setting-up a feed value which is lower than the combined width of the groove and pile-up. The tip trace in this case is illustrated in Fig. 7(a) and it uses a $15 \mathrm{~nm}$ feed, which is approximately half of the groove period measured in the previous section. The machined arrays of grooves are shown in Fig. 7(b). It can be seen from this figure that the resulting topography of the array is similar to that of Fig. 5(a) because a $30 \mathrm{~nm}$ wavelength was also obtained. Thus, it can be said that one visible groove consists of two "designed" proximity grooves. A possible hypothesis for explaining this somewhat unexpected result is that the small feed used here may lead to the tip sliding down the face of the pile-up when hitting it. This phenomenon may happen repeatedly during the entire length of the linear motion of the probe in the anti-parallel direction of the previously formed groove. The fact that the average depth of the grooves in the array produced in this way is larger than that achieved with the $30 \mathrm{~nm}$ feed reported 
earlier would tend to support this hypothesis. Indeed, the amplitude of arrays of grooves was approximately $10 \mathrm{~nm}$ with the feed of $15 \mathrm{~nm}$ (see Fig. 7(b). It is also observed that the array of grooves cross-section was a quasi-sinusoid pattern that could be fitted very well with a sinusoidal function of period $30 \mathrm{~nm}$ (see "Fitting curve" in the plot of Fig 7(b)). In addition, it should be mentioned that the ratio of wavelength to amplitude was three, whereas it was six for the array of grooves shown earlier in Fig. 5(a) with a feed of $30 \mathrm{~nm}$. As a result, a ratio of larger feature sizes could be generated by this "combination writing" method. Thus, a decreased feed allows the formation of an array of grooves with a larger amplitude. This also suggests that the feed could be used to control the amplitude/wavelength ratio.

(a)

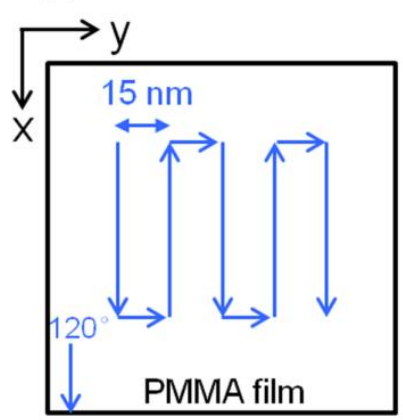

(b)

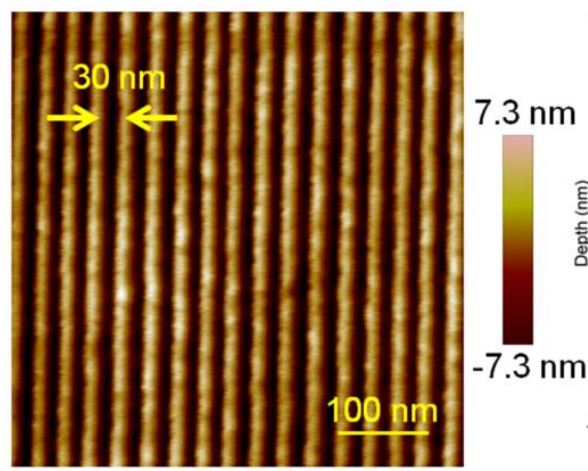

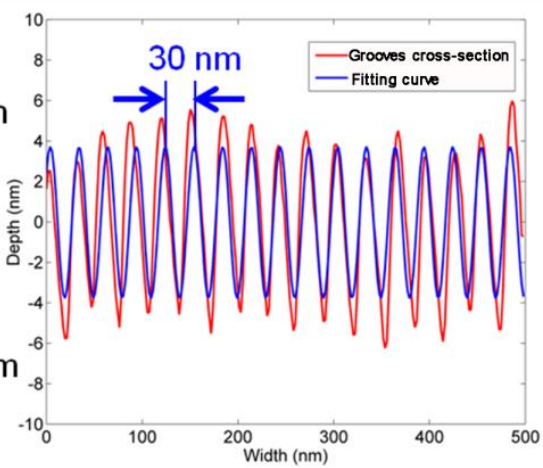

Fig. 7 (a) Schematic of the tip trace with a $15 \mathrm{~nm}$ feed in the $120^{\circ}$ direction and (b) resulting $30 \mathrm{~nm}$ wavelength array of grooves.

\subsection{Controlled nanodot fabrication with arbitrary orientation}

As discussed above, the "combination writing" method is used to obtain arrays of regular grooves on a PMMA thin film. Thus, by processing two or more layers oriented at different angles using this approach, it may be possible to generate an array of nanodots. Fig. 8 shows such results, where layers of $30 \mathrm{~nm}$ wavelength arrays of grooves oriented at different angles are used to fabricate nanodots. The scratching parameters are identical for each scratching direction. The drive amplitude ratio $\left(V_{w} / V_{r}\right)$ was five and the scratching speed was $0.5 \mu \mathrm{m} / \mathrm{s}$. Checkerboard nanodots are 
obtained with a two-step scratching method in the $30^{\circ}$ and $120^{\circ}$ directions (see Fig. 8(a)). The nanodot length is equal to the $30 \mathrm{~nm}$ wavelength of the array of grooves, and the corresponding density could be as high as $1.3 \times 10^{9} \mathrm{dots} / \mathrm{mm}^{2}$. A fast Fourier transform (FFT) of the nanodot array indicates high periodicity in both horizontal and vertical directions. Diamond-shaped nanodots are generated in the $90^{\circ}$ and $150^{\circ}$ directions, as shown in Fig. 8(b). A three-step scratching method in the $30^{\circ}, 90^{\circ}$, and $150^{\circ}$ directions is utilized to fabricate hexagonal nanodots, as shown in Fig. 8(c). The densities are $9.6 \times 10^{8}$ dots $/ \mathrm{mm}^{2}$ and $1.9 \times 10^{9}$ dots $/ \mathrm{mm}^{2}$, respectively. The height of the nanodots was less than $10 \mathrm{~nm}$ in all of the 3 arrays shown in Fig. 8. Thus, the orientation of nanodots can be controlled by a combination of different scratching directions, and various forms of nanodots can be fabricated with controlled height and length. It is also worth stating that the method presented here avoids adjusting the scratching parameters between the different layers to achieve specific feature sizes, as was done in [16]. In addition, the method does not require the sample orientation to be changed for producing such arrays of nanodots as was implemented in [25]. Besides, the density of the nanodots achieved here was larger than that of the results reported in [16] and [25]. 

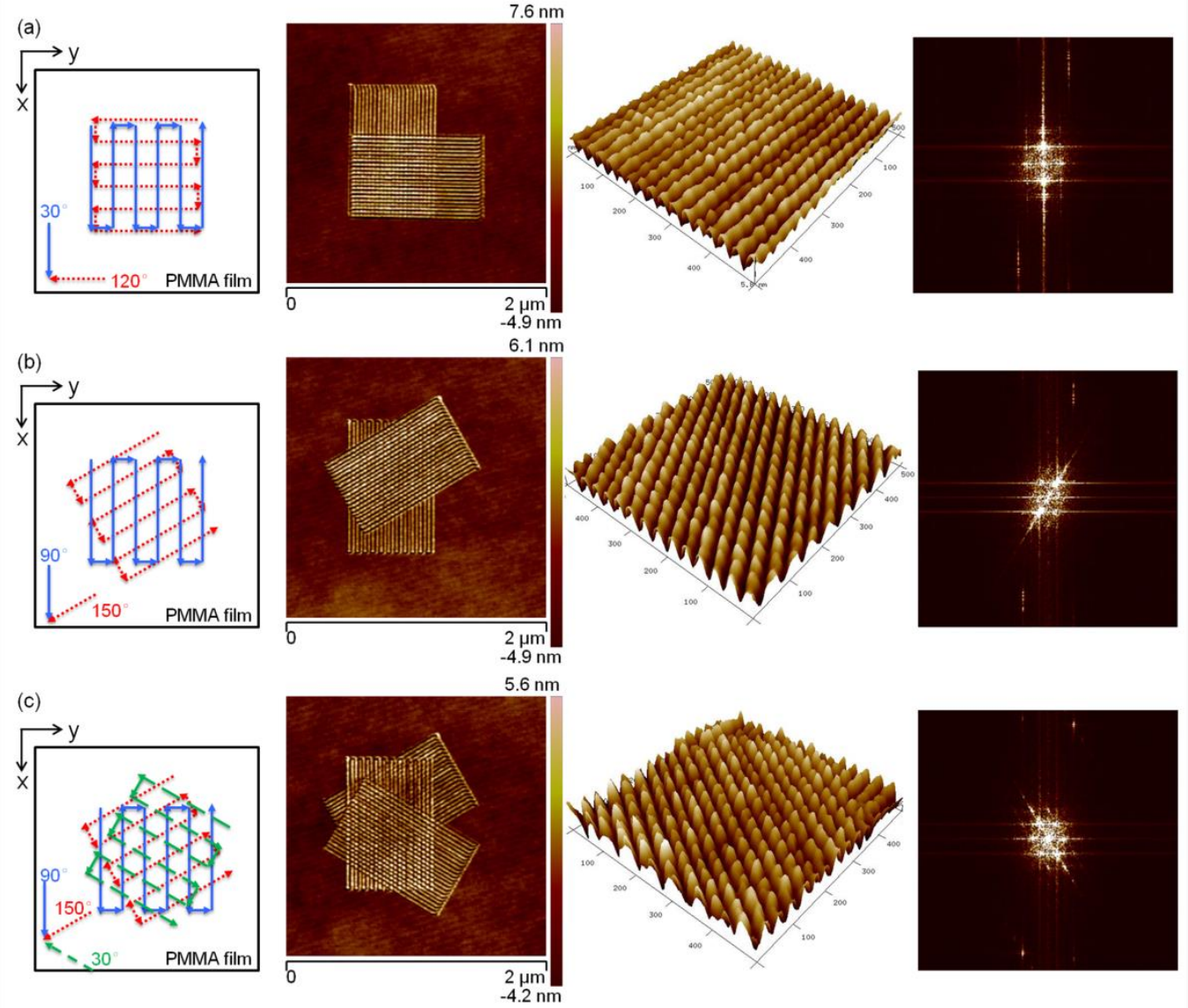

Fig. 8 Nanodot fabrication. FFT images (far right) of the AFM morphologies. (a) Checkerboard nanodots fabricated by cross scratching in the $30^{\circ}$ and $120^{\circ}$ directions. (b) Diamond-shaped nanodots fabricated in the $90^{\circ}$ and $150^{\circ}$ directions. (c) Hexagonal nanodots fabricated in the $30^{\circ}$, $90^{\circ}$, and $150^{\circ}$ directions.

\section{Conclusions}

In this study, utilizing the DPL technique, an asymmetric tip was used to fabricate grooves on a PMMA thin film. The machined results were investigated with regard to effects of scratching directions on feature sizes. In addition, obtained periodic nanostructures were derived from machining arrays of grooves. The tip trace was optimized to promote the quality of the periodic nanostructures. The following conclusions could be obtained. 
(1) The geometry of the tip contributed to the shape of the pile-up. However, groove features, such as depth, mouth width, period, and pile-up height, are almost independent from the scratching direction, except for the direction parallel to and away from the long axis of the cantilever.

(2) For identical scratching directions, the wavelength of parallel grooves is dependent on the feed, or the groove period. For achieving proximity grooves, the feed had to be less than $40 \mathrm{~nm}$. The ratio of wavelength to amplitude was six. The so-called "scan-scratch" tip trace strategy can be used to limit processing time and tip wear. In this case, adjacent grooves follow anti-parallel directions. The machined results reveal that a combination of two arrays of grooves yields irregular patterns. Unpredicted shapes in arrays of grooves were caused by the pile-up shape generated in different directions. Thus, "combination writing" method was proposed to fabricate arrays of grooves. In this case, the wavelength of the array of grooves produced was $30 \mathrm{~nm}$, which was twice the feed value, and the wavelength/amplitude ratio was three.

(3) Finally, with the "combination writing" method in the $30^{\circ}$ and $120^{\circ}$ directions, nanodot checkerboards were obtained with density as high as $1.3 \times 10^{9}$ dots $/ \mathrm{mm}^{2}$. Diamond-shaped and hexagonal nanodots arrays were obtained with densities of $9.6 \times 10^{8} \mathrm{dots} / \mathrm{mm}^{2}$ and $1.9 \times 10^{9}$ $\operatorname{dots} / \mathrm{mm}^{2}$, respectively.

\section{Acknowledgements}

The authors gratefully acknowledge the financial support of the Foundation for the National Natural Science Foundation of China (51675134), Innovative Research Groups of the National Nat ural Science Foundation of China (51521003), Self-Planned Task (SKLRS201606B) of State Key Laboratory of Robotics and System (HIT), the Program for New Century Excellent Talents in Univ ersity (NCET-11-0812), and the National Program for Support of Top-notch Young Professors.

\section{References}


[1] Li JF, Huang YF, Ding Y, Yang ZL, Li SB, Zhou XS, et al., Shell-isolated nanoparticle-enhanced Raman spectroscopy, Nature. 464(2010)392-5.

[2] Choi CH, Lee DJ, Sung J-H, Lee MW, Lee S-G, Park S-G, et al., A study of AFM-based scratch process on polycarbonate surface and grating application, Applied Surface Science, 256(2010)7668-71.

[3] Liu L, Khan HA, Li J, Hillier AC, Lu M, A strain-tunable nanoimprint lithography for linear variable photonic crystal filters, Nanotechnology. 27(2016)295301.

[4] Wang J, Huang L, Yuan L, Zhao L, Feng X, Zhang W, et al., Silver nanostructure arrays abundant in sub-5nm gaps as highly Raman-enhancing substrates, Applied Surface Science. 258(2012)3519-23.

[5] García-Vidal FJ, Pendry JB, Collective theory for surface enhanced Raman scattering, Physical Review Letters. 77(1996)1163-6.

[6] Schweikart A, Fery A, Controlled wrinkling as a novel method for the fabrication of patterned surfaces, Microchimica Acta. 165(2009)249-63.

[7] Kim HS, Crosby AJ, Solvent-responsive surface via wrinkling instability, Adv Mater. 23(2011)4188-92.

[8] Cai S, Breid D, Crosby AJ, Suo Z, Hutchinson JW, Periodic patterns and energy states of buckled films on compliant substrates, Journal of the Mechanics and Physics of Solids. 59(2011)1094-114.

[9] Meng J, Xie J, Han X, Lu C, Surface wrinkling on polydopamine film, Applied Surface Science. 371(2016)96-101.

[10] Gao T, Xu Z, Fang F, Gao W, Zhang Q, Xu X, High performance surface-enhanced Raman scattering substrates of Si-based Au film developed by focused ion beam nanofabrication, Nanoscale research letters. 7(2012)1.

[11] Yan Y, Geng Y, Hu Z, Recent advances in AFM tip-based nanomechanical machining, International Journal of Machine Tools and Manufacture. 99(2015)1-18. 
[12] Yu J, Kim SH, Yu B, Qian L, Zhou Z, Role of tribochemistry in nanowear of single-crystalline silicon, ACS Appl Mater Interfaces. 4(2012)1585-93.

[13] Guo J, Yu B, Chen L, Qian L, Nondestructive nanofabrication on Si(100) surface by tribochemistry-induced selective etching, Sci Rep. 5(2015)16472.

[14] Geng Y, Yan Y, Xing Y, Zhang Q, Zhao X, Hu Z, Effect of cantilever deformation and tip-sample contact area on AFM nanoscratching, Journal of Vacuum Science \& Technology B: Microelectronics and Nanometer Structures. 31(2013)061802.

[15] Al-Musawi RS, Brousseau EB, Geng Y, Borodich FM, Insight into mechanics of AFM tip-based nanomachining: bending of cantilevers and machined grooves, Nanotechnology. 27(2016)385302.

[16] Yan Y, Sun Y, Li J, Hu Z, Zhao X, Controlled nanodot fabrication by rippling polycarbonate surface using an AFM diamond tip, Nanoscale research letters. 9(2014)1-7.

[17] Sun Y, Yan Y, Hu Z, Zhao X, Yan J, 3D polymer nanostructures fabrication by AFM tip-based single scanning with a harder cantilever, Tribology International. 47(2012)44-9.

[18] Wang Y, Hong X, Zeng J, Liu B, Guo B, Yan H, AFM Tip Hammering Nanolithography, Small. 5(2009)477-83.

[19] Kunze U, Klehn B, Plowing on the Sub-50nm Scale: Nanolithography Using Scanning Force Microscopy, Advanced Materials. 11(1999)1473-5.

[20] Heyde M, Rademann K, Cappella B, Geuss M, Sturm H, Spangenberg T, et al., Dynamic plowing nanolithography on polymethylmethacrylate using an atomic force microscope, Review of Scientific Instruments. 72(2001)136.

[21] Yan Y, He Y, Geng Y, Hu Z, Zhao X, Characterization study on machining PMMA thin-film using AFM tip-based dynamic plowing lithography, Scanning.38 (2016)612-618.

[22] Shim W, Brown KA, Zhou X, Rasin B, Liao X, Schmucker AL, et al., Plow and ridge nanofabrication, Small. 9(2013)3058-62. 
[23] Cappella B, Sturm H, Weidner S, Breaking polymer chains by dynamic plowing lithography, Polymer. 43(2002)4461-6.

[24] Tseng AA, Shirakashi J-i, Nishimura S, Miyashita K, Notargiacomo A, Scratching properties of nickel-iron thin film and silicon using atomic force microscopy, Journal of Applied Physics. 106(2009)044314.

[25] Tseng AA, Kuo C-FJ, Jou S, Nishimura S, Shirakashi J-i, Scratch direction and threshold force in nanoscale scratching using atomic force microscopes, Applied Surface Science. 257(2011)9243-50. 


\section{Figure captions}

Fig1. (a) Schematic of grooves with pile-up fabrication on a PMMA thin film using DPL; (b) edgeand face-writing directions; (c) geometry of a silicon tip; (d) AFM images of grooves obtained from different scanning directions.

Fig. 2 Morphology and cross-sections (red lines) of grooves fabricated with (a) pile-up accumulated at $90^{\circ}$ along one side, and (b) pile-up accumulated at $0^{\circ}$ along both sides.

Fig. 3 Groove feature sizes: (a) width, (b) period, (c) depth, and (d) height for different scratching directions.

Fig. 4 (a) Schematic of tip trace, (b) morphology of circles with 1, 2, 3, 4, and 5- $\mu$ m radii and the circle with the radius of $1 \mu \mathrm{m}$.

Fig. 5 Schematic of tip traces and AFM images of arrays of grooves obtained in the direction of $90^{\circ}$ for a feed of (a) $30 \mathrm{~nm}$ and (b) $40 \mathrm{~nm}$.

Fig. 6 Schematic of tip trace and corresponding machined results. (a) and (e) a "scan-scratch" tip trace in two directions. Arrays of grooves obtained with a $30-\mathrm{nm}$ feed with a direction of (b) $30^{\circ}$ (c) $120^{\circ}$ (f) $60^{\circ}$ and (g) $150^{\circ}$. (d) and (h) Irregular patterns from the combination of arrays of grooves with two-step scratching process.

Fig. 7 (a) Schematic of the tip trace with a $15-\mathrm{nm}$ feed in the $120^{\circ}$ direction.(b) 30 -nm wavelength array of grooves.

Fig. 8 Nanodot fabrication. FFT images (far right) of the AFM morphologies. (a) Checkerboard nanodots fabricated by cross scratching in the $30^{\circ}$ and $120^{\circ}$ directions. (b) Diamond-shaped nanodots fabricated in the $90^{\circ}$ and $150^{\circ}$ directions. (c) Hexagonal nanodots fabricated in the $30^{\circ}$, $90^{\circ}$, and $150^{\circ}$ directions. 